\title{
Effects of antihypertensive drugs losartan and levamlodipine besylate on insulin resistance in patients with essential hypertension combined with isolated impaired fasting glucose
}

\author{
Wei-yin Xiao ${ }^{1}$, Ning Ning ${ }^{1}$, Ming-Hong Tan ${ }^{1}$, Xue-Shu Jiang ${ }^{1}$, Liang Zhou ${ }^{2}$, Ling Liu ${ }^{2}$, Dong Yi ${ }^{2}$ and Ping Wei ${ }^{1}$
}

The objective of this study was to observe the antihypertensive effect of losartan and levamlodipine besylate on insulin resistance in patients with essential hypertension (EH) combined with isolated impaired fasting glucose (i-IFG). Patients $(n=244)$ were randomly assigned to losartan potassium tablets (50-100 mg per day) or levamlodipine besylate tablets (2.5-5.0 mg per day) for intensive antihypertensive treatment with no lifestyle interventions for 3 years. The changes in fasting plasma glucose, fasting insulin (FINS) and insulin sensitivity index (ISI) from before to after treatment were observed. Blood pressure (BP) in each group was significantly reduced by treatment $(P<0.05)$. After 12 months of treatment, the FINS level in the losartan potassium group was significantly decreased and ISI was significantly increased compared with before treatment $(P<0.05)$ and compared with the levamlodipine besylate group $(P<0.05)$. After 24 and 36 months of treatment, FINS was significantly decreased and ISI was significantly improved in both groups compared with baseline $(P<0.05)$, and there was no difference between the groups $(P>0.05)$. The incidence of new-onset diabetes mellitus was not significantly different between two groups. The antihypertensive effect of losartan and levamlodipine besylate could amoliorate insulin resistance in patients with EH combined with i-IFG. The improvement of insulin resistance by losartan potassium at 12 months might be better than that by levamlodipine besylate; however, after $\mathbf{2 4}$ and $\mathbf{3 6}$ months of follow-up, both agents significantly alleviated insulin resistance. These results suggest that the effects of these two drugs on insulin resistance are not significantly different.

Hypertension Research (2016) 39, 321-326; doi:10.1038/hr.2015.155; published online 14 January 2016

Keywords: essential hypertension; insulin resistance; isolated impaired fasting glucose

\section{INTRODUCTION}

The most recent epidemiological survey data of diabetes mellitus (DM) in China showed that the incidence of prediabetes, including impaired fasting glucose (i-IFG), was 50.1\%. ${ }^{1}$ IFG is an independent risk factor for DM. The risk of progression into DM in the IFG population is two to three times more than that in the general population. ${ }^{2,3}$ With the increase in fasting glucose, the risk of cardiovascular diseases, ischemic heart diseases, cardiac infarction and thrombotic stroke also gradually increase. Therefore, IFG could be used as a predictive factor for stroke and coronary arteriosclerotic heart diseases. ${ }^{4}$ Chen et al. ${ }^{5}$ analyzed indicators such as blood pressure (BP) and fasting plasma glucose (FPG) in 1026 patients with essential hypertension (EH) and showed that the incidence of IFG in hypertensive patients was $30.5 \%$, which was higher compared with the incidence of IFG $(21.25 \%)$ in normal individuals reported by Yang et al. ${ }^{6}$
These findings showed that hypertensive patients are more likely to have combined IFG than normal individuals. When combined with impaired glucose metabolism, the cardiovascular complications in hypertensive patients significantly increase, and this situation is closely associated with the mortality rate. ${ }^{7,8}$

In the process of reducing $\mathrm{BP}$, some angiotensin II receptor blockers can also reduce the progression of impaired glucose tolerance (IGT) into DM. ${ }^{9}$ However, it is unknown whether the benefit is from the drop in BP or the type of drugs. There are few studies on the outcome and intervention of patients with EH combined with i-IFG. Therefore, it is important to explore methods to inhibit or reduce the progression of EH combined with i-IFG into DM, to observe the effect of the hypertension on the outcome of i-IFG and to confirm whether the improvement of abnormal blood glucose is caused by antihypertensive drugs or the reduction of BP. Our findings should contribute to the

${ }^{1}$ Department of Endocrinology, Southwest Hospital, Third Military Medical University, ChongQing, China and ${ }^{2}$ Department of Health Statistics, Third Military Medical University, ChongQing, China

Correspondence: Professor P Wei, Department of Endocrinology, Southwest Hospital, 30 Gaotanyan Street, Chongqing 400038, China.

E-mail:wp301@sina.com

or Professor D Yi, Department of Health Statistics, Third Military Medical University, 30 Gaotanyan Street, Chongqing 400038, China.

E-mail: yd_house@hotmail.com

Received 5 January 2015; revised 12 May 2015; accepted 28 August 2015; published online 14 January 2016 
effective prevention or delay of type $2 \mathrm{DM}$ in many prediabetic patients.

\section{METHODS}

\section{Study design}

This is a randomized, double-blind, prospective cohort design, 36-month study. Subjects were randomly assigned into two groups. One group was administered angiotensin receptor blockers (ARBs) for antihypertension and losartan potassium at 50 or $100 \mathrm{mg}$. The other group was administered calcium channel blockers for antihypertension and levamlodipine besylate at 2.5 or $5 \mathrm{mg}$. We required that BP be reduced below 130/80 $\mathrm{mm} \mathrm{Hg}$. The BP of subjects was monitored in the long term, and observation indicators were re-examined every 12 months.

During the treatment period, there is no lifestyle interventions that was prescribed. All enrolled subjects were followed up by telephone once every month to help them understand how to measure blood glucose and BP by themselves, and remind them of the educational points mentioned above. All enrolled patients came to the clinic of our department for follow-up every 6 months.

\section{Subjects}

Patients with EH combined with i-IFG in the outpatient Department of Endocrinology and the physical examination center who met the following inclusion criteria were enrolled: (1) age between 18 and 70 years; (2) $i$-IFG criteria: ${ }^{10}$ they received at least two fasting glucose (FG) examinations on different days in the Clinical Laboratory of our hospital, and the results showed $5.6 \mathrm{mmol}^{-1}<\mathrm{FPG}<7 \mathrm{mmol}^{-1}$ and postprandial 2-h plasma glucose (2hPG) $<7.8 \mathrm{mmol} \mathrm{1}^{-1}$; (3) hypertension criteria: ${ }^{11}$ the BP was the average of three measurements of BP in the right arm after sitting still for 5 min using a cuff sphygmomanometer, which conformed to the standards formulated in the 2010 Chinese guidelines for the management of hypertension: systolic BP (SBP) $\geqslant 140 \mathrm{~mm} \mathrm{Hg}(1 \mathrm{~mm} \mathrm{Hg}=0.133 \mathrm{kPa})$ and diastolic BP $(\mathrm{DBP}) \geqslant 90 \mathrm{~mm} \mathrm{Hg}$; (4) patients had not used antihypertensive drugs within the previous 2 weeks; (5) patients who would like to come back for follow-up in the next 3 years.

Exclusion criteria: (1) patients who were incapable or unwilling to provide written informed consent; (2) evidence of liver disease (alanine aminotransferase or aspartate aminotransferase greater than twice the normal upper limit) or kidney disease (serum creatinine $>95 \mu \mathrm{mol}^{-1}$ ); (3) secondary hypertension, urinary tract infection, renal artery stenosis, hyperkalemia, pregnancy, lactation, recent cerebral hemorrhage or cerebral infarction, or severe heart failure; (4) use of hypoglycemic medication or insulin in the previous 5 years; (5) allergy to the drugs in this study; (6) participants who refused to come back to the hospital for follow-up.

This study protocol was approved by the Ethics Committee of the Southwest Hospital of The Third Military University (2011 RSKY No. 25) and was registered in the Chinese Clinical Trial Registry Center (TRC.11001653). Written informed consents was obtained from all participants.

\section{Observation indicators}

The major observation indicators were SBP, fasting insulin (FINS), insulin sensitivity index (ISI), FPG, 2-h insulin (2hINS) and 2hPG. The minor indicators were DBP, glycohemoglobin (HbAlC), body mass index (BMI), total cholesterol, total triglycerides (TGs), low-density lipoprotein cholesterol and high-density lipoprotein cholesterol (HDL-C).

The above indicators were all tested in the Clinical Laboratory and the Department of Nuclear Medicine in our hospital. At the first visit, all patients had fasted for 10-12 h for standard oral glucose tolerance tests and insulin release tests. Plasma glucose (PG) was determined by the glucose oxidase method and insulin was determined by radioimmunoassay. Other biochemical indicators were measured with enzymatic methods. The degree of insulin resistance (IR) is presented as ISI, where ISI $=$ In $(1 /(\mathrm{FPG} \times \mathrm{FINS})){ }^{12}$ Dyslipidemia was diagnosed according to the dyslipidemia indicators in the diagnostic standards of metabolic syndrome proposed by the International Diabetes Federation in 2005: TG $\geqslant 1.7 \mathrm{mmol}^{-1}$ and HDL-C $<1.03 \mathrm{mmoll}^{-1}$ (males) or HDL-C $<1.29 \mathrm{mmol} \mathrm{l}^{-1}$ (females). ${ }^{13}$ BMI $\geqslant 24 \mathrm{~kg} \mathrm{~m}^{-2}$ was overweight and BMI $\geqslant 28 \mathrm{~kg} \mathrm{~m}^{-2}$ was obesity. ${ }^{14}$

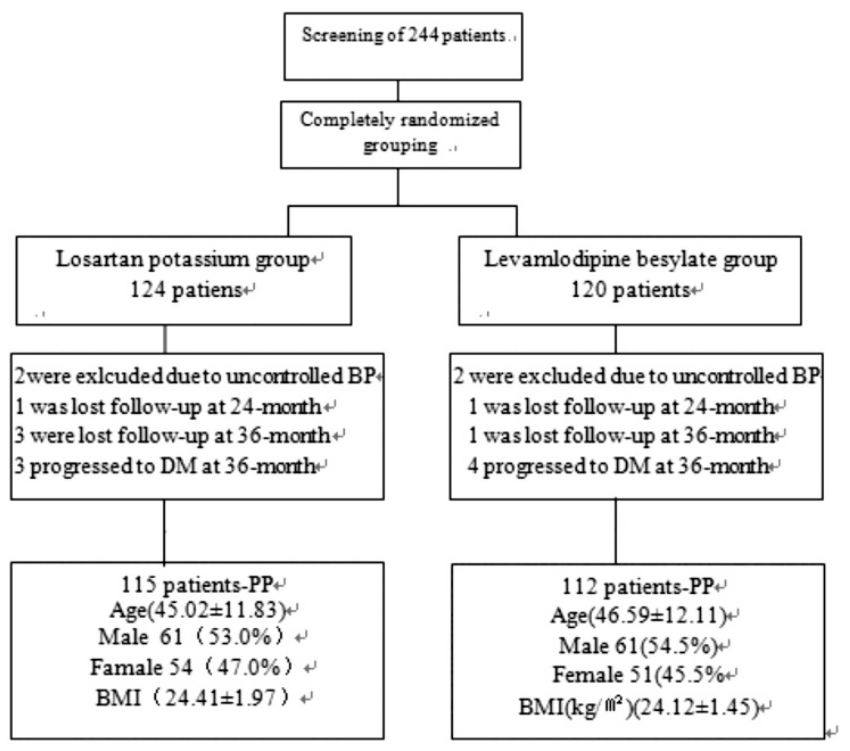

Figure 1 Enrollment of patients, loss of patients and demographic characteristics.

\section{Statistical analysis}

Statistical analysis was performed using SPSS 18.0 software (IBM SPSS, Chicago, IL, USA). All variables were checked for normal distribution before data analysis. The data are expressed as arithmetic means \pm s.d. for normally distributed variables. According to the intention-to-treat design of the study, all subjects (completers and noncompleters) were included in the final analysis. The description of efficacy results mainly targeted per-protocol population, and safety analysis targeted all enrolled populations. The pairwise comparison between groups and within groups was conducted using the repeated measurement method. The influence of the baseline was calibrated using the covariance method. $P$-value $<0.05$ after correction was considered significant.

\section{RESULTS}

The enrollment situation and demographic characteristics

A total of 244 patients were randomly enrolled (losartan potassium: $n=124$; levamlodipine besylate: $n=120$ ). Four were excluded because their BP control did not reach the standard (two in the losartan potassium group and two in the levamlodipine besylate group). Two were lost to follow-up after 24 months (one in the losartan potassium group and one in the levamlodipine besylate group). Four were lost to follow-up after 36 months (three in the losartan potassium group and one in the levamlodipine besylate group). After 36 months of followup, seven patients had progressed to DM (three in the losartan potassium group and four in the levamlodipine besylate group). A total of 227 patients completed the 36 months of follow-up (115 in the losartan potassium group and 112 in the levamlodipine besylate group). These 277 patients were used as the per-protocol data analysis set, and the efficacy of the medications was analyzed (Figure 1).

The differences in age, gender and BMI between the losartan potassium group and the levamlodipine besylate group did not reach statistical significance. Among all patients, 26.4\% $(n=60)$ had severe IR, $63 \%(n=143)$ were overweight or obese and $79.3 \%$ had abnormal TG and/or HDL-C $(n=180)$. During the randomized grouping, the comparison of all indicators in demographics did not show obvious differences (Figure 1). 


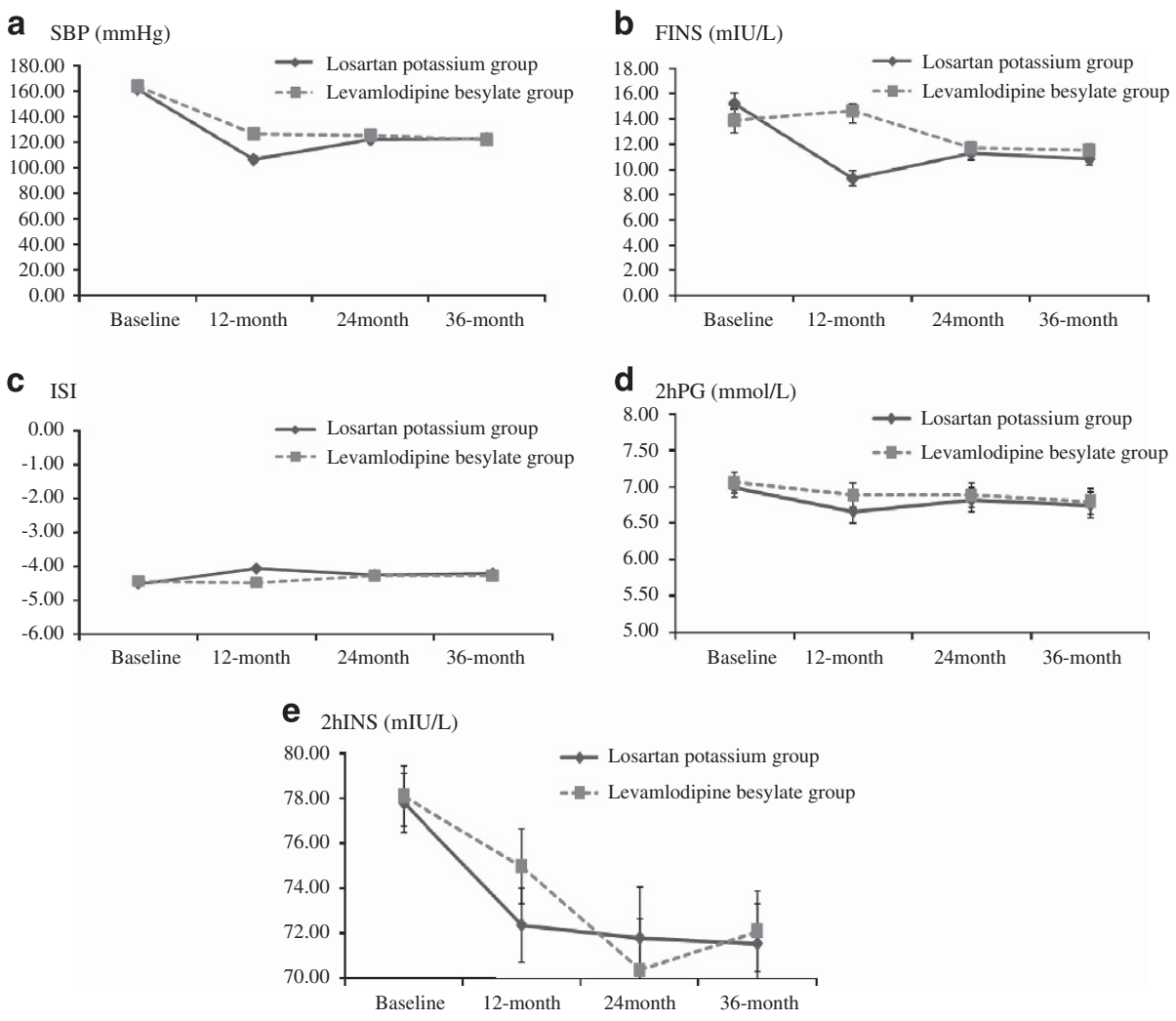

Figure 2 Time series of changing trend of major efficacy indicators. A full color version of this figure is available at the Hypertension Research journal online.

\section{Analysis of efficacy}

FINS and ISI. After 12 months of follow-up, FINS decreased (12-month FINS 9.33 (95\% confidence interval (CI): 8.73, 9.93)) and ISI increased (12-month ISI -4.06 (95\% CI: $-4.11,-4.02))$ in the losartan potassium group; the differences were statistically significant compared with baseline. FINS and ISI in the levamlodipine besylate group remained similar to baseline. The differences between these two groups were statistically significant at 12 months. After 24 and 36 months of follow-up, compared with baseline, FINS in both groups decreased (losartan potassium group: 24-month FINS 11.27 (95\% CI: 10.76, 11.79) and 36-month FINS 10.89 (95\% CI: 10.39, 11.39); levamlodipine besylate group: 24-month FINS 11.72 (95\% CI: $11.19,12.24)$ and 36-month FINS 11.55 (95\% CI: 11.04,12.05)) and ISI increased (losartan potassium group: 24-month ISI -4.24 (95\% CI: $-4.29,-4.19)$ and 36 -month ISI -4.20 (95\% CI: $-4.25,-4.16$ ); levamlodipine besylate group: 24-month ISI -4.27 (95\% CI: -4.31 , -4.22), 36-month ISI -4.25 (95\% CI: $-4.30,-4.21)$ ), there was no significant difference between these two groups. After 24 and 36 months of visit, compared with 12 months of follow-up, FINS increased (24-month FINS 11.27 (95\% CI: 10.76, 11.79) and 36-month FINS 10.89 (95\% CI: 10.39, 11.39)) and ISI decreased (24-month ISI $-4.24(95 \%$ CI: $-4.29,-4.19)$ and 36 -month ISI $-4.20(95 \%$ CI: $-4.25,-4.16))$ in the losartan potassium group, and FINS lowered (24-month FINS 11.72 (95\% CI: 11.19, 12.24) and 36-month FINS 11.55 (95\% CI: $11.04,12.05)$ ) and ISI heightened ( 24-month ISI - 4.27 (95\% CI: $-4.31,-4.22)$ and 36-month ISI -4.25 (95\% CI: -4.30 , $-4.21)$ ) in the levamlodipine besylate group (Table 2 and Figure 2).

Blood pressure. Compared with baseline, BP in both groups significantly decreased. At 12 months of follow-up, compared with SBP in the levamlodipine besylate group (12-month SBP 126.67 (95\% CI: $124.18,129.16)), \mathrm{SBP}$ in the losartan potassium group significantly decreased (12-month SBP 106.57 (95\% CI: 104.12, 109.03)). There was no significant differences observed in BP at other visit time between two groups (Table 2 and Figure 2).

FPG, 2hINS, HbAlc and 2hPG. Compared with baseline, FPG had not changed significantly after 36 months in either group. In contrast, 2hINS in both groups decreased (losartan potassium group: 36-month 2hINS 71.58 (95\% CI: 69.77, 73.38); levamlodipine besylate group: 36-month 2hINS 71.96 (95\% CI: 70.14, 73.79)), and there was no difference between these two groups. At 36 months of follow-up, HbAlC (36-month HbAlc 5.88 (95\% CI: 5.80, 5.96)) decreased in the losartan potassium group compared with baseline. HbAlc was not significantly different between groups at all follow-up times. At 12 months of follow-up, $2 \mathrm{hPG}$ in the losartan potassium group decreased compared with baseline (12-month 2hPG 6.65 (95\% CI: $6.48,6.81)) .2 \mathrm{hPG}$ was not significantly different between groups at all follow-up times (Table 2 and Figure 2).

BMI, TG, total cholesterol, HDL cholesterol and low-density lipoprotein cholesterol. BMI stayed similar to baseline in both groups. After 36 months of follow-up, TG in the losartan potassium group was similar to baseline. TG in the levamlodipine besylate group increased at 12 and 36 months (12-month TG 2.25 (95\% CI: 2.10, 2.39) and 36-month TG 2.22 (95\% CI: 2.08, 2.37)), the differences were statistically significant. HDL-C in the losartan potassium group decreased at 24 and 36 months compared with baseline (24-month HDL-C 1.27 (95\% CI: 1.21, 1.32) and 36-month HDL-C 1.25 (95\% CI: $1.19,1.32)$ ). HDL-C in the levamlodipine besylate group decreased 
at every visit time compared with baseline (12-month HDL-C 1.26 (95\% CI: 1.20, 1.32), 24-month HDL-C 1.25 (95\% CI: 1.19, 1.31) and 36-month HDL-C 1.28 (95\% CI: 1.21, 1.34)), the differences were statistically significant. Total cholesterol and low-density lipoprotein cholesterol stayed similar to baseline in both groups. Blood lipids were not significantly different between groups at any time (Table 2 and Figure 2).

\section{Correlational analysis}

FINS and SBP showed a positive correlation $(r=0.997, P=0.03)$, whereas ISI and SBP showed a negative correlation $(r=-0.992$, $P=0.008)$ in the losartan potassium group. FINs and SBP had a weak positive correlation $(r=0.462, P=0.538)$, whereas ISI and SBP had a weak negative correlation $(r=-0.496, P=0.504)$ in the levamlodipine besylate group. $2 \mathrm{hPG}$ and $2 \mathrm{hINS}$ had a weak positive correlation with SBP in each group (Table 3).

\section{Safety and adverse events}

The drug tolerance in these two groups was good, and there was no adverse event. Seven patients progressed to DM (three in the losartan potassium group $(2.61 \%)$ and four in the levamlodipine besylate group $(3.57 \%))$. There was no significant difference between these two rates $\left(\chi^{2}\right.$ test: $\left.P=0.176\right)$.

\section{CONCLUSIONS}

In recent years, many clinical and basic studies have been published on the clinical pathological status of IFG. Epidemiological studies indicate that IFG and IGT exist simultaneously in only a small population, and these two conditions have different effects on IR. ${ }^{2}$ Abdul-Ghani et al. ${ }^{15}$ studied populations with impaired IFG or IGT, and showed that in contrast to the occurrence of IR in the muscle of IGT patients, IFG patients had severe IR in the liver and mild IR in the muscle, which was associated with the timing of insulin secretion impairment. ${ }^{16}$ The risks of IFG and IGT to progress to DM are similar and are closely associated with cardiovascular risk factors such as hypertension and hyperlipemia. ${ }^{16}$ A study in South Korea showed that in primary hypertension patients, as FPG increases, the risk of the onset of cardiovascular diseases, ischemic heart diseases, myocardial infarction and thrombotic stroke also increase. IFG could be used as a predictive factor for stroke and coronary atherosclerotic heart diseases. ${ }^{17}$ The conclusion of the study published in 2011 by the largest-scale DM prevention study in the world, NAVIGATOR, also suggested that IFG could predict the occurrence of atrial fibrillation. ${ }^{18}$ The populationbased Rotterdam study stated that IFG was closely associated with the decrease of carotid arterial distensibility in elderly. ${ }^{19}$ The FPG levels of hypertensive patients selected in this study all reached the IFG diagnostic standard (Table 1).

The correlation between hypertension and IFG and whether there is a common pathogenesis is hot spot. The Tecumseh study found that with increased BP combined with changes in blood glucose and insulin, patients with mild hypertension show increased sympathetic nerve excitability and impaired glucose metabolism. ${ }^{20}$ Forty to fifty percent of hypertensive patients have IR. Hemodynamic changes might be the pathophysiological basis of IR in hypertensive patients. The increase in BP combined with changes in blood glucose and insulin might be associated with increased sympathetic nerve excitability and changes to hemorheology. Hypertension combined with impaired glucose metabolism could increase cardiovascular complications double to threefold. ${ }^{7,8,21,22}$ Among the patients with $\mathrm{EH}$ combined with i-IFG in this study, 26.4\% $(n=60)$ had severe IR. This was lower compared with the percentage in previous reports,
Table 1 Clinical characteristics of the patients in the two treatment groups at baseline

Losartan potassium group Levamlodipine besylate group $(\mathrm{n}=115)$ $(\mathrm{n}=112)$

\begin{tabular}{lcc}
\hline SBP $(\mathrm{mm} \mathrm{Hg})$ & $161.24(159.42,163.07)$ & $163.80(161.95,165.66)$ \\
FPG $\left(\mathrm{mmol} \mathrm{I}^{-1}\right)$ & $6.31(6.22,6.40)$ & $6.40(6.31,6.49)$ \\
FINS $\left(\mathrm{mlU} \mathrm{I}^{-1}\right)$ & $15.12(14.28,15.96)$ & $13.86(13.01,14.71)$ \\
$2 \mathrm{hINS}\left(\mathrm{mlU} \mathrm{I}^{-1}\right)$ & $77.92(76.58,79.27)$ & $78.19(76.83,79,55)$ \\
ISI & $-4.51(-4.57,-4.45)$ & $-4.43(-4.49,-4.37)$ \\
2hPG (mmol I-1) & $6.98(6.83,7.12)$ & $7.04(6.89,7.18)$ \\
$\mathrm{HbAlc}\left(\%, \mathrm{mmol} \mathrm{I}^{-1}\right)$ & $6.17(6.04,6.30)$ & $6.07(5.94,6.21)$ \\
& $44.03(42.58,45.47)$ & $42.92(41.45,44.39)$ \\
DBP (mm Hg) & $100.65(99.22,102.09)$ & $102.93(101.48,104.38)$ \\
TG (mmol I-1) & $2.08(1.99,2.17)$ & $1.92(1.83,2.01)$ \\
TC (mmol I-1) & $4.54(4.46,4.67)$ & $4.55(4.41,4.69)$ \\
HDL-C (mmol I-1) & $1.39(1.34,1.45)$ & $1.46(1.40,1.51)$ \\
LDL-C (mmol I-1) & $2.80(2.68,2.90)$ & $2.79(2.68,2.90)$ \\
\hline
\end{tabular}

Abbreviations: BMI, body mass index; DBP, diastolic blood pressure; FPG, fasting plasma glucose; FINS, fasting insulin; HbAlc, glycohemoglobin; HDL-C, high-density lipoprotein cholesterol; 2hINS, 2-hour insulin; 2hPG, 2-h plasma glucose; ISI, insulin sensitivity index; LDL-C, low-density lipoprotein cholesterol; SBP, systolic blood pressure; TC, total cholesterol; $\mathrm{TG}$, total triglyceride.

which might be associated with the certain people. Nonetheless, the results showed a significant rate of IR in hypertensive patients who have i-IFG (Table 1).

The VALUE (Valsartan Antihypertensive Long-term Use Evaluation) study stated that blood glucose was the most important predictive factor for new-onset DM in hypertensive patients with high cardiovascular risk. ${ }^{23}$ However, the impact of different kinds of antihypertensive drugs on incident diabetes is controversial, a network meta-analysis stated that the association of antihypertensive drugs with incident $\mathrm{DM}$ is therefore lowest for $\mathrm{ARB}$ and the angiotensinconverting enzyme inhibitors followed by calcium channel blocker and placebo, $\beta$-blockers and diuretics in rank order. ${ }^{24}$ Andraws and Brown $^{25}$ performed a meta-analysis on the Life study (losartan), CHARM-Overall study (candesartan), SCOPE study (candesartan), ALPINE study (candesartan) and VALUE study (valsartan). The results suggested that the ARBs used in the above studies reduced the risk of the occurrence of DM by $27 \%$, and their effect was similar to that of the angiotensin-converting enzyme inhibitors. ${ }^{25}$ The 2010 NAVIGATOR study concluded that ARBs could significantly reduce FPG and 2hPG in IGT populations. ${ }^{9}$ Taguchi et al. ${ }^{26}$ also found that irbesartan have anti-inflammatory and antioxidative effects, as well as beneficial effects on glucose in hypertensive outpatients. Therefore, this effect of inhibiting new-onset DM was thought to originate specifically from the function of ARBs; however, the specific mechanism underlying the reduction of DM risk by ARBs is not clear. Compared with the ARB drug olmesartan, the calcium channel blocker drug manidipine might improve statin-related IR in hypertensive patients who have IFG. ${ }^{27}$ Another study also found that calcium channel blocker drug cilnidipine can improve glucose and insulin sensitivity in diet-induced obese mice. ${ }^{28}$ The subjects in this study were patients with $\mathrm{EH}$ combined with i-IFG. They were randomly assigned into two groups, the losartan potassium group and the levamlodipine besylate group. The results showed that after 12 months of follow-up, FINS decreased and ISI increased in the losartan potassium group, suggesting that losartan potassium might have the ability to improve IR in a short time. However, SBP in the 


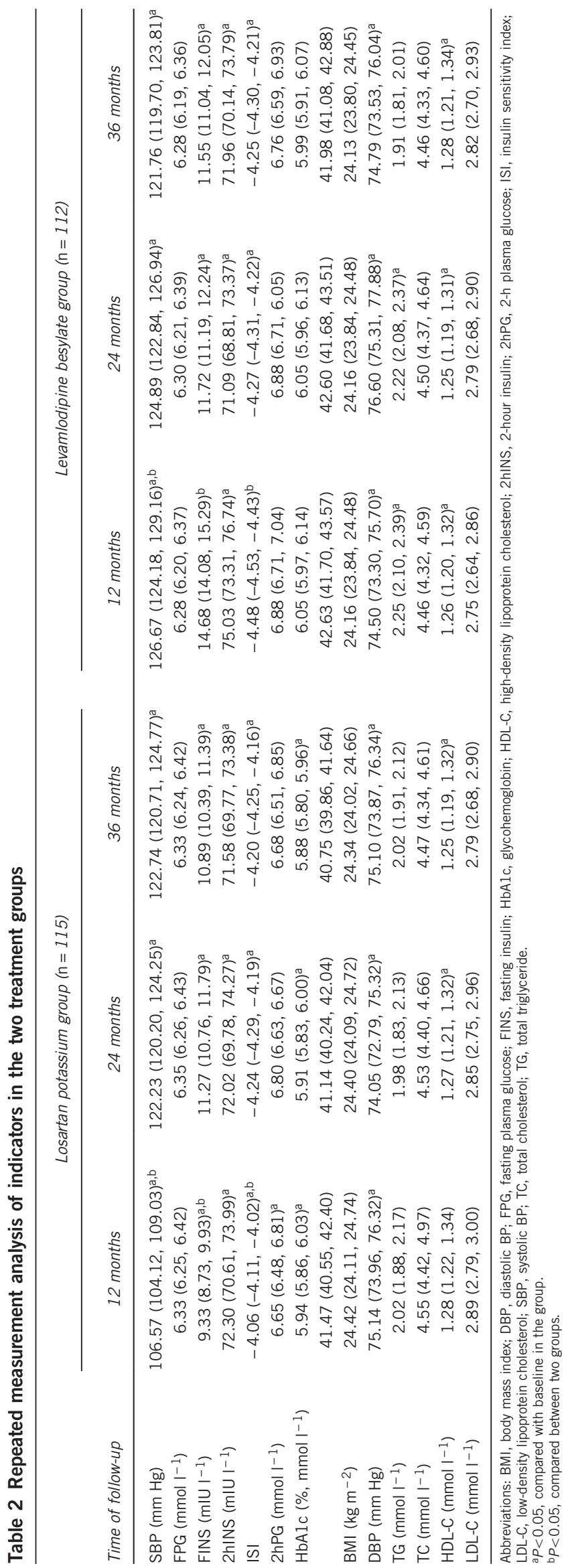

Table 3 Correlational analysis between SBP and major efficacy indicators

\begin{tabular}{lccccc}
\hline & \multicolumn{2}{c}{ Losartan potassium group } & & \multicolumn{2}{c}{ Levamlodipine besylate group } \\
\cline { 2 - 3 } \cline { 6 - 6 } & R-value & P-value & & R-value & P-value \\
\hline FINS & 0.997 & 0.03 & & 0.462 & 0.538 \\
ISI & -0.992 & 0.008 & & -0.496 & 0.504 \\
2hPG & 0.942 & 0.058 & & 0.913 & 0.087 \\
2hINS & 0.916 & 0.084 & & 0.881 & 0.119 \\
\hline
\end{tabular}

Abbreviations: FINS, fasting insulin; 2hINS, 2-hour insulin; 2hPG, 2-h plasma glucose; ISI, insulin sensitivity index; SBP, systolic blood pressure.

losartan potassium group significantly decreased at 12 months compared with that in the levamlodipine besylate group. We speculate that the differences in FINS and ISI between these two groups might come from the differences in SBP. At 24 and 36 months of follow-up, FINS and ISI were not significantly different between groups; this trend was correlated with the changes in SBP (Tables 2 and 3 and Figure 2).

Diabetes management guidelines state that ARBs and angiotensinconverting enzyme inhibitors are the cornerstone drugs for treating patients with type $2 \mathrm{DM}$ accompanied by hypertension. ${ }^{10}$ In addition to effectively reducing $\mathrm{BP}, \mathrm{ARBs}$ and angiotensin-converting enzyme inhibitors also improve glucose metabolism, and there are other antihypertensive drugs such as hydrochlorothiazide and $\beta$-blockers may aggravate IR and have adverese effect on glycometabolism. ${ }^{29-31}$ However, the selection of antihypertensive drugs in the 2013 European Society of Hemotology/European Society of Cardiology Guidelines for the management of arterial hypertension clearly shows that the main benefit of antihypertensive treatment comes from the reduction of BP itself and is largely independent of the choice of drug. Meta-analyses have shown that the claims of superiority of some specific drugs are largely caused by study biases. The largest meta-analysis did not show any difference in the clinical effects of the different drugs. In addition, the Guidelines state that the claimed AR activation effect of telmisartan has not been confirmed. ${ }^{32}$ These results indicate that the differentiation between drugs of the same category is mainly based on speculation rather than mechanism; therefore, it is recommended that DM patients can use all types of antihypertensive drugs. For this study, we selected the population with EH combined with i-IFG. We showed that as BP decreased, IR were significantly improved. The results show that the improvement might be associated with the type of antihypertensive drug only at 12 months. Therefore, we speculate that the impaired blood glucose and IR in the population of patients with $\mathrm{EH}$ combined with i-IFG might come from the increased BP itself. Successful control of BP could improve the IR of this population and reduce the occurrence of new-onset DM.

IR is common in patients with EH combined with i-IFG and is usually combined with impaired metabolism such as obesity and dyslipidemia. With no lifestyle intervention recommended, antihypertensive treatment of losartan potassium and levamlodipine besylate can improve the IR in this population. The improvement of IR by 12 months of losartan potassium might be better than that of levamlodipine besylate. However, at 24 and 36 months of follow-up, IR in these two groups were both significantly improved. The incidence of new-onset DM was not significantly different between these two groups, suggesting that the improvement of IR in this population was mainly due to the reduction of BP itself. The effects of 
these two antihypertensive drugs on IR were not significantly different. Therefore, no matter which of these two antihypertensive drugs is used, long-term successful control of BP becomes the major intervention goal of this population, and it can be effective to prevent the occurrence of new-onset DM.

\section{CONFLICT OF INTEREST}

The authors declare no conflict of interest.

\section{ACKNOWLEDGEMENTS}

The study was supported by clinical project of Third Military Medical University (2010XLC04) and was supported by three grants from the Natural Science Foundation of China (Nos 81172773, 81202286 and 81473068). The guarantor's of this article is PW.

Author contributions: W-yX enrolled patients, performed the experiments and wrote the manuscript; PW conducted the study, supervised the project and revised the manuscript; DY designed the study and analyzed the data; NN, M-HT and X-SJ contributed in data collection; LZ and LL helped in software and statistic.

1 Xu Y, Wang L, He J, Bi Y, Li M, Wang T, Wang L, Jiang Y, Dai M, Lu J, Xu M, Li Y, Hu N, Li J, Mi S, Chen CS, Li G, Mu Y, Zhao J, Kong L, Chen J, Lai S, Wang W, Zhao W, Ning G. Prevalence and control of diabetes in Chinese adults. JAMA 2013; 310: 948-959.

2 Abdul-Ghani MA, Lyssenko V, Tuomi T, DeFronzo RA, Groop L. Fasting vs. postload plasma glucose concentration and the risk for future type 2 diabetes: results from the Botnia Study. Diabetes Care 2009; 32: 281-286.

3 Wannamethee SG, Shaper AG, Lennon L, Morris RW. Metabolic syndrome vs. Framingham Risk Score for prediction of coronary heart disease, stroke, and type 2 diabetes mellitus. Arch Intern Med 2005; 165: 2644-2650.

4 Park C, Guallar E, Linton JA, Lee DC, Jang Y, Son DK, Han EJ, Baek SJ, Yun YD, Jee SH, Samet JM. Fasting glucose level and the risk of incident atherosclerotic cardiovascular diseases. Diabetes Care 2013; 36: 1988-1993.

5 Chen YZ, Hua Q, Liu RK. Investigation and analysis of the prevalence and risk factors for impaired fasting glucose in outpatients with essential hypertension. Zhonghua Nei Ke Za Zhi 2008; 47: 731-734.

6 Yang ZJ, Yang WY, Xiao JZ, Li GW, Wang Y. Impact of lowering the cut-point for impaired fasting glucose on the distribution of impaired glucose regulation subcategories in Chinese adult population. Zhonghua Yi Xue Za Zhi 2004; 84: 1773-1776.

7 Ferrannini E, Cushman WC. Diabetes and hypertension: the bad companions. Lancet 2012; 380: 601-610.

8 El-Atat F, McFarlane SI, Sowers JR. Diabetes, hypertension, and cardiovascular derangements: pathophysiology and management. Curr Hypertens Rep 2004; 6: 215-223.

9 Bloch MJ, Basile JN. In navigator, the angiotensin receptor blocker valsartin modestly reduced the progression to type 2 diabetes but had no effect on cardiovascular events in higher-risk patients with impaired glucose tolerance. J Clin Hypertens (Greenwich) 2010; 12: 881-885.

10 American Diabetes Association. Standards of medical care in diabetes-2014. Diabetes Care 2014; 37 (Suppl 1): S14-S80.

11 Liu LS. 2010 Chinese guidelines for the management of hypertension. Zhonghua Xin Xue Guan Bing Za Zhi 2011; 39: 579-615.

12 Matthews DR, Hosker JP, Rudenski AS, Naylor BA, Treacher DF, Turner RC. Homeostasis model assessment: insulin resistance and beta-cell function from fasting plasma glucose and insulin concentrations in man. Diabetologia 1985; 28: 412-419.

13 Guerrero-Romero F, Rodriguez-Moran M. Concordance between the 2005 International Diabetes Federation definition for diagnosing metabolic syndrome with the National Cholesterol Education Program Adult Treatment Panel III and the World Health Organization definitions. Diabetes Care 2005; 28: 2588-2589.
$14 \mathrm{Li} X Y$, Jiang Y, Hu N, Li YC, Zhang M, Huang ZJ, Zhao WH. Prevalence and characteristic of overweight and obesity among adults in China, 2010. Zhonghua $Y u$ Fang Yi Xue Za Zhi 2012; 46: 683-686.

15 Abdul-Ghani MA, Tripathy D, DeFronzo RA. Contributions of beta-cell dysfunction and insulin resistance to the pathogenesis of impaired glucose tolerance and impaired fasting glucose. Diabetes Care 2006; 29: 1130-1139.

16 Unwin N, Shaw J, Zimmet P, Alberti KG. Impaired glucose tolerance and impaired fasting glycaemia: the current status on definition and intervention. Diabetes Med 2002; 19: 708-723.

17 Kim HK, Kim CH, Kim EH, Bae SJ, Choe J, Park JY, Park SW, Yun YD, Baek SJ, Mok Y, Jee SH. Impaired fasting glucose and risk of cardiovascular disease in Korean men and women: the Korean Heart Study. Diabetes Care 2013; 36: 328-335.

18 Latini R, Staszewsky L, Sun JL, Bethel MA, Disertori M, Haffner SM, Holman RR, Chang F, Giles TD, Maggioni AP, Rutten GE, Standl E, Thomas L, Tognoni G, Califf RM, McMurray $\mathrm{JJ}$. Incidence of atrial fibrillation in a population with impaired glucose tolerance: the contribution of glucose metabolism and other risk factors. A post hoc analysis of the Nateglinide and Valsartan in Impaired Glucose Tolerance Outcomes Research trial. Am Heart J 2013; 166: 935-940.

19 van Popele NM, Elizabeth HA, Mattace-Raso FU, Bots ML, van der Kuip DA, Reneman RS, Hoeks AP, Hofman A, Grobbee DE, Witteman JC. Impaired fasting glucose is associated with increased arterial stiffness in elderly people without diabetes mellitus: the Rotterdam Study. J Am Geriatr Soc 2006; 54: 397-404.

20 Julius S, Jamerson K, Mejia A, Krause L, Schork N, Jones K. The association of borderline hypertension with target organ changes and higher coronary risk. Tecumseh Blood Pressure study. JAMA 1990; 264: 354-358.

21 Bonora E, Targher G, Zenere MB, Saggiani F, Cacciatori V, Tosi F, Travia D, Zenti MG, Branzi P, Santi L, Muggeo M. Relationship between fasting insulin and cardiovascular risk factors is already present in young men: the Verona Young Men Atherosclerosis Risk Factors Study. Eur J Clin Invest 1997; 27: 248-254.

22 Arnlov J, Pencina MJ, Nam BH, Meigs JB, Fox CS, Levy D, D'Agostino RB, Vasan RS. Relations of insulin sensitivity to longitudinal blood pressure tracking: variations with baseline age, body mass index, and blood pressure. Circulation 2005; 112: 1719-1727.

23 Aksnes TA, Kjeldsen SE, Rostrup M, Omvik P, Hua TA, Julius S. Impact of new-onset diabetes mellitus on cardiac outcomes in the Valsartan Antihypertensive Long-term Use Evaluation (VALUE) trial population. Hypertension 2007; 50: 467-473.

24 Elliott WJ, Meyer PM. Incident diabetes in clinical trials of antihypertensive drugs: a network meta-analysis. Lancet 2007; 369: 201-207.

25 Andraws R, Brown DL. Effect of inhibition of the renin-angiotensin system on development of type 2 diabetes mellitus (meta-analysis of randomized trials). Am J Cardiol 2007; 99: 1006-1012.

26 Taguchi I, Toyoda S, Takano K, Arikawa T, Kikuchi M, Ogawa M, Abe S, Node K, Inoue T. Irbesartanan angiotensin receptor blocker, exhibits metabolic, anti-inflammatory and antioxidative effects in patients with high-risk hypertension. Hypertens Res 2013; 36: 608-613.

27 Liberopoulos EN, Moutzouri E, Rizos CV, Barkas F, Liamis G, Elisaf MS. Effects of manidipine plus rosuvastatin vs. olmesartan plus rosuvastatin on markers of insulin resistance in patients with impaired fasting glucose, hypertension, and mixed dyslipidemia. J Cardiovasc Pharmacol Ther 2013; 18: 113-118.

28 Ueno D, Masaki T, Gotoh K, Chiba S, Kakuma T, Yoshimatsu H. Cilnidipine regulates glucose metabolism and levels of high-molecular adiponectin in diet-induced obese mice. Hypertens Res 2013; 36: 196-201.

29 Eriksson JW, Jansson PA, Carlberg B, Hagg A, Kurland L, Svensson MK, Ahlstrom H, Strom C, Lonn L, Ojbrandt K, Johansson L, Lind L. Hydrochlorothiazide, but not candesartan, aggravates insulin resistance and causes visceral and hepatic fat accumulation: the mechanisms for the diabetes preventing effect of Candesartan (MEDICA) Study. Hypertension 2008; 52: 1030-1037.

30 Cooper-DeHoff RM, Wen S, Beitelshees AL, Zineh I, Gums JG, Turner ST, Gong Y, Hall K, Parekh V, Chapman AB, Boerwinkle E, Johnson JA. Impact of abdominal obesity on incidence of adverse metabolic effects associated with antihypertensive medications. Hypertension 2010; 55: 61-68.

31 Guo Q, Mori T, Jiang Y, Hu C, Ohsaki Y, Yoneki Y, Nakamichi T, Ogawa S, Sato H, Ito S. Losartan modulates muscular capillary density and reverses thiazide diureticexacerbated insulin resistance in fructose-fed rats. Hypertens Res 2012; 35: 48-54.

32 ESH/ESC Task Force for the Management of Arterial Hypertension. 2013 Practice guidelines for the management of arterial hypertension of the European Society of Hypertension (ESH) and the European Society of Cardiology (ESC): ESH/ESC Task Force for the Management of Arterial Hypertension. J Hypertens 2013; 31: 1925-1938. 